\title{
Numerical analyses of deflagration initiation by a hot jet
}

\author{
I. Iglesias ${ }^{\mathrm{a}}$, M. Vera ${ }^{\mathrm{a}}$, A.L. Sánchez ${ }^{\mathrm{a} *}$ and A. Liñán ${ }^{\mathrm{b}}$
}

\begin{abstract}
Numerical simulations of axisymmetric reactive jets with one-step Arrhenius kinetics are used to investigate the problem of deflagration initiation in a premixed fuel-air mixture by the sudden discharge of a hot jet of its adiabatic reaction products. For the moderately large values of the jet Reynolds number considered in the computations, chemical reaction is seen to occur initially in the thin mixing layer that separates the hot products from the cold reactants. This mixing layer is wrapped around by the starting vortex, thereby enhancing mixing at the jet head, which is followed by an annular mixing layer that trails behind, connecting the leading vortex with the orifice rim. A successful deflagration is seen to develop for values of the orifice radius larger than a critical value $a_{\mathrm{c}}$ in the order of the flame thickness of the planar deflagration $\delta_{\mathrm{L}}$. Introduction of appropriate scales provides the dimensionless formulation of the problem, with flame initiation characterised in terms of a critical Damköhler number $\Delta_{\mathrm{c}}=\left(a_{\mathrm{c}} / \delta_{\mathrm{L}}\right)^{2}$, whose parametric dependence is investigated. The numerical computations reveal that, while the jet Reynolds number exerts a limited influence on the criticality conditions, the effect of the reactant diffusivity on ignition is much more pronounced, with the value of $\Delta_{c}$ increasing significantly with increasing Lewis numbers Le. The reactant diffusivity affects also the way ignition takes place, so that for reactants with Le $\gtrsim 1$ the flame develops as a result of ignition in the annular mixing layer surrounding the developing jet stem, whereas for highly diffusive reactants with Lewis numbers sufficiently smaller than unity combustion is initiated in the mixed core formed around the starting vortex. The analysis provides increased understanding of deflagration initiation processes, including the effects of differential diffusion, and points to the need for further investigations incorporating detailed chemistry models for specific fuel-air mixtures.
\end{abstract}

Keywords: deflagration; ignition; transient hot jet; starting vortex; differential diffusion

\section{Introduction}

It is known that the starting jet formed by the sudden discharge of hot reaction products into a fuel-air mixture can serve to initiate the combustion process [1]. The problem has important implications in connection with the transport, handling and storage of fuels, particularly hydrogen, and may also find application in engine ignition systems [2].

The starting jet formed by the sudden discharge of hot products into a quiescent atmosphere through a circular orifice in a plane wall shows a characteristic structure including a leading vortex ring followed by a slender jet stem. The associated jet dynamics has been studied in detail for constant density configurations [3,4] and also for 
low-Mach-number gaseous jets with variable temperature [5]. The vortex sheet shed as the boundary layer separates at the orifice rim rolls up spirally to form a toroidal vortex that travels downstream, wrapping around the fluid surface separating the hot jet from the outer cold reactants. As a result of the roll-up, a mixed core of reactants and combustion products is seen to form at the jet head, providing a precursor ignition kernel where chemical reactions are enabled by the relatively high temperature and radical content. Mixing also occurs in the annular mixing layer surrounding the jet stem that trails behind the leading vortex. This mixing layer may be strained due to the appearance of secondary vortices when the jet Reynolds number is sufficiently large.

The combustion mode initiated by the hot jet depends fundamentally on the value of the discharge orifice radius $a$, to be compared with the characteristic scales of premixed combustion, with relevant lengths being the characteristic detonation cell size $\delta_{d}$ and the characteristic deflagration thickness

$$
\delta_{\mathrm{L}}=\frac{\lambda_{\mathrm{b}} / c_{p}}{\rho_{\mathrm{u}} U_{\mathrm{L}}}
$$

defined from the corresponding laminar burning rate $\rho_{\mathrm{u}} U_{\mathrm{L}}$, with $\rho_{\mathrm{u}}$ and $U_{\mathrm{L}}$ being the values of unburnt gas density and freely-propagating planar-flame velocity and $\lambda_{\mathrm{b}}$ and $c_{p}$ representing the thermal conductivity and specific heat at constant pressure of the burnt mixture. For given conditions of pressure, composition and initial temperature, there exists a critical orifice radius $a_{\mathrm{c}} \sim \delta_{\mathrm{L}}$, with $\delta_{\mathrm{L}} \sim 3 \times 10^{-4} \mathrm{~m}$ at normal atmospheric conditions, below which ignition is precluded, whereas for larger values of $a>a_{\mathrm{c}}$ a deflagration forms following the jet discharge. The initiation of a detonation requires much larger values of $a$, in the order of $\delta_{\mathrm{d}} \sim 10^{-1} \mathrm{~m} \mathrm{[6].}$

The present paper deals with hot jets of transverse size much smaller than $\delta_{\mathrm{d}}$, of interest for pulsed combustion jet systems [2]. This problem is also relevant in connection with the design of flameproof enclosures for electrical equipment [7], whose orifices and gaps must be sufficiently small to ensure that, if a reactive mixture enters the enclosure and becomes ignited, the jet of combustion products escaping outside is unable to ignite the surrounding gas mixture. Typical hole/gap sizes for these systems lie in the millimeter to submillimeter range, so that a deflagration is seen to form following a successful ignition event. Of utmost importance for safety applications is the critical size below which combustion transmission is precluded. In the case of axysimmetric jets, values of critical orifice radii for propane-air mixtures, measured in a specific experimental facility, are available [8]. Also, the problem has been studied recently for hydrogen-air mixtures by a combination of experimental and numerical methods [9], providing increased understanding of the influence of the jet temperature and mixing process on ignition occurrence. A related problem also addressed in the past $[10,11]$ is that of jet diffusion flames formed by the sudden discharge of a fuel jet into an oxidiser atmosphere.

As in previous studies [8,9], the objective of the present work is to contribute understanding to the problem of hot-jet initiation of a deflagration. To that end, numerical integrations of the conservation equations are performed to describe the combustion response for different values of the flow parameters and their influence on the value of the critical orifice radius $a_{\mathrm{c}}$. The jet scales are used in formulating the transient jet discharge in dimensionless form, yielding a problem that is integrated numerically to determine the critical Damköhler number $\left(a_{\mathrm{c}} / \delta_{\mathrm{L}}\right)^{2}$, above which a deflagration forms, as a function of a reduced set of governing parameters. The results are very dependent on the diffusivity 
of the deficient reactant through its associated Lewis number, whereas dependences on additional parameters including the jet Reynolds number are found to be much weaker.

For subcritical cases, a weakly-reactive jet flow is formed for large times, with mixing of the hot products with the ambient gas and chemical reaction occurring without deflagration formation. The resulting flow is slender and steady, and can therefore be described with the boundary-layer approximation. The integration of the resulting parabolic problem, independent of the jet Reynolds number, provides a simpler alternative way to compute critical conditions for ignition. When the reactant diffusivity is sufficiently small, the results obtained are in close agreement with those of transient jet computations, but for larger reactant diffusivities, including in particular that of lean hydrogen mixtures, significant differences appear. Reasons for these differences are to be discussed below.

\section{Formulation}

Consider a jet of hot combustion products discharging at the initial instant with uniform velocity $U_{j}$ through an orifice of radius $a$ into a stagnant unburnt mixture at temperature $T^{\prime}=T_{\mathbf{u}}$ and density $\rho^{\prime}=\rho_{\mathbf{u}}$, containing a mass fraction $Y=Y_{\mathbf{u}}$ of reactant. A single irreversible reaction $\mathrm{R} \rightarrow \mathrm{P}$ will be assumed to describe the conversion of reactant into products, with $q$ denoting the amount of heat released per unit mass of reactant consumed. Correspondingly, $T_{\mathrm{b}}=T_{\mathrm{u}}+q Y_{\mathrm{u}} / c_{p}$ is the adiabatic flame temperature. In typical cases, the resulting temperature increment $\alpha=\left(T_{\mathrm{b}}-T_{\mathrm{u}}\right) / T_{\mathrm{b}}$ takes on values in the range $0.7 \leq$ $\alpha \leq 0.85$.

The rate $w$ of reactant mass consumed per unit volume and unit time will be assumed to be given by the Arrhenius law $w=\rho Y B \exp \left[-E /\left(R^{\circ} T\right)\right]$ with $B$ being a constant frequency factor and $E$ and $R^{\circ}$ denoting the activation energy and the universal gas constant. The value of the laminar burning rate obtained at leading order in the asymptotic limit of large activation energy [1],

$$
\rho_{\mathrm{u}} U_{\mathrm{L}}=\left\{\frac{2 \mathrm{Le} \lambda_{\mathrm{b}} \rho_{\mathrm{b}} B \exp \left[-E /\left(R^{\circ} T_{\mathrm{b}}\right)\right]}{c_{p} \beta^{2}}\right\}^{1 / 2},
$$

will be used below in formulating the problem in terms of the Damköhler number

$$
\Delta=\left(\frac{a}{\delta_{\mathrm{L}}}\right)^{2}
$$

with the flame thickness $\delta_{\mathrm{L}}$ defined for a given reactive mixture in Equation (1). Here, $\rho_{\mathrm{b}}$ is the burnt gas density, $\beta=\left(T_{\mathrm{b}}-T_{\mathrm{u}}\right) E /\left(R^{\circ} T_{\mathrm{b}}^{2}\right)$ is the Zeldovich number, and Le is the reactant Lewis number. A successful deflagration initiation will be seen to occur for values of $a$ larger than a critical value $a_{\mathrm{c}}$ in the order of the flame thickness, i.e. values of the Damköhler number larger than a critical value $\Delta_{c}=\left(a_{c} / \delta_{L}\right)^{2}$ of order unity.

Compressibility effects are neglected in the analysis, as it is appropriate when the overpressure in the neighbouring compartment containing the products is small compared with the existing ambient pressure, giving values of $U_{j}$ much smaller than the velocity of sound. The associated jet Reynolds number

$$
\operatorname{Re}=\frac{\rho_{\mathrm{b}} U_{j} a}{\mu_{\mathrm{b}}},
$$


where $\mu_{\mathrm{b}}$ is the viscosity of the burnt gases, will be assumed to be moderately large. Note that, since $\operatorname{Re} \sim\left(U_{j} / U_{\mathrm{L}}\right)\left(a / \delta_{\mathrm{L}}\right)$, with $a \sim \delta_{\mathrm{L}}$ being the distinguished limit for deflagration initiation, this last assumption implies jet velocities $U_{j} \sim \operatorname{Re} U_{\mathrm{L}} \gg U_{\mathrm{L}}$. Axial symmetry with no azimuthal motion will be assumed in the computations, an approach that precludes the description of the helical modes often observed at sufficiently large values of Re [12].

To write the problem in dimensionless form, the initial jet velocity $U_{j}$ is used as scale for the axial and radial velocity components, $u$ and $v$, the jet radius $a$ is used to scale the axial and radial coordinates $x$ and $r$, and the characteristic time $a / U_{j}$ is used to scale the time $t$. The pressure differences from the far-field ambient value, $p^{\prime}-p_{a}^{\prime}$, are scaled with the dynamic pressure $\rho_{\mathrm{b}} U_{j}^{2}$ to give the variable $p=\left(p^{\prime}-p_{a}^{\prime}\right) /\left(\rho_{\mathbf{b}} U_{j}^{2}\right)$. Normalised forms $\varphi=Y / Y_{\mathrm{u}}$ and $\Theta=\left(T^{\prime}-T_{\mathrm{u}}\right) /\left(T_{\mathrm{b}}-T_{\mathrm{u}}\right)$ are introduced for the reactant mass fraction and the temperature. In terms of these dimensionless variables, the conservation equations for the transient axisymmetric jet reduce to

$$
\begin{aligned}
\frac{\partial u}{\partial x}+\frac{1}{r} \frac{\partial}{\partial r}(r v) & =0 \\
\frac{\mathrm{D} u}{\mathrm{D} t} & =-\frac{\partial p}{\partial x}+\frac{1}{\operatorname{Re}}\left[\frac{1}{r} \frac{\partial}{\partial r}\left(r \frac{\partial u}{\partial r}\right)+\frac{\partial^{2} u}{\partial x^{2}}\right] \\
\frac{\mathrm{D} v}{\mathrm{D} t} & =-\frac{\partial p}{\partial r}+\frac{1}{\operatorname{Re}}\left[\frac{\partial}{\partial r}\left(\frac{1}{r} \frac{\partial}{\partial r}(r v)\right)+\frac{\partial^{2} v}{\partial x^{2}}\right] \\
\operatorname{Re} \frac{\mathrm{D} \varphi}{\mathrm{D} t} & =\frac{1}{\operatorname{Le}} \frac{1}{\operatorname{Pr}}\left[\frac{1}{r} \frac{\partial}{\partial r}\left(r \frac{\partial \varphi}{\partial r}\right)+\frac{\partial^{2} \varphi}{\partial x^{2}}\right]-\Omega \\
\operatorname{Re} \frac{\mathrm{D} \Theta}{\mathrm{D} t} & =\frac{1}{\operatorname{Pr}}\left[\frac{1}{r} \frac{\partial}{\partial r}\left(r \frac{\partial \Theta}{\partial r}\right)+\frac{\partial^{2} \Theta}{\partial x^{2}}\right]+\Omega,
\end{aligned}
$$

where

$$
\Omega=\frac{\Delta \beta^{2}}{2 \operatorname{Pr} \mathrm{Le}} \varphi \exp \left[-\frac{\beta(1-\Theta)}{1-\alpha(1-\Theta)}\right]
$$

and

$$
\frac{\mathrm{D}}{\mathrm{D} t}=\frac{\partial}{\partial t}+u \frac{\partial}{\partial x}+v \frac{\partial}{\partial r}
$$

represent the dimensionless reaction rate and material derivative, respectively, and $\mathrm{Pr}$ is the constant Prandtl number. As can be seen, variations of density and transport properties arising from temperature and composition changes have been neglected in writing Equations (5)-(9). Although this simplifying assumption introduces a certain degree of inaccuracy in quantitative predictions of $\Delta_{c}$, the associated errors are not expected to be very large. This shall be confirmed below in the framework of the boundary-layer integrations of Section 4.2, which include a number of computations with variable density and variable transport properties. 
The problem is to be integrated for $t>0$ with boundary conditions

$$
x=0: \quad \begin{cases}r \leq 1: & u=1, v=\varphi=\Theta-1=0 \\ r>1: & u=v=\partial \varphi / \partial x=\partial \Theta / \partial x=0\end{cases}
$$

corresponding to a jet of combustion products issuing at temperature $T=T_{\mathrm{b}}$ from an orifice in a non-permeable adiabatic wall. For $x>0$, the symmetry condition

$$
r=0: \quad \partial u / \partial r=v=\partial \varphi / \partial r=\partial \Theta / \partial r=0
$$

is imposed at the axis, whereas the boundary conditions as $r^{2}+x^{2} \rightarrow \infty$ include the ambient values $\varphi-1=\Theta=0$, along with an outflow condition for the velocity and pressure at the external boundaries of the domain, which was selected to be a cylindrical surface of radius $r_{\max } \gg 1$ extending downstream to $x_{\max } \gg 1$. The values $r_{\max }=200$ and $x_{\max }=500$ were employed in most computations, except for some slowly developing ignition events, such as those shown below for the less diffusive reactant with $\mathrm{Le}=2$, for which larger values $r_{\max }=300$ and $x_{\max }=800$ were required.

Initial values $u=v=\Theta=0$ and $\varphi=1$ are used for the temperature and reactant mass fraction at $t=0$. Note that, for the incompressible flow assumed here, the outer stagnant fluid is impulsively set into motion at the initial instant to adjust to the boundary condition for the velocity imposed at the orifice, so that the velocity distribution for $t \ll 1$ corresponds to the potential flow generated by a uniform distribution of volumetric sources located at the orifice [5].

\section{Results of numerical integrations}

Integration of Equations (5)-(9) with the given initial and boundary conditions provides the evolution of the flow for a given set of values of the flow parameters, namely Re, Le, $\Delta, \operatorname{Pr}, \alpha$ and $\beta$. The conservation equations were discretised following a cell-centred finite-volume procedure. A second-order upwind scheme was used to evaluate the face values of the variables for the convective terms, while second-order central differences were used for the diffusive terms. The pressure-velocity coupling was achieved with an algorithm of the SIMPLE family. The time discretisation was implicit and first-order accurate. The computational domain was discretised in a block-structured grid. As a representative example, for the equidiffusional case $\mathrm{Le}=1$ with $\mathrm{Re}=100$ the initial grid consisted of a square grid with $\delta x=\delta r=0.5$ covering the whole domain plus a very refined grid in a region of dimensions $(0 \leq x \leq 1.5,0 \leq r \leq 1.25)$ around the jet exit, with minimum spacing $\delta x=\delta r=7.8125 \times 10^{-3}$. This original grid was dynamically adapted every 10 time steps to extend the refined grid up to the region where the gradient of $\Theta$ reached a threshold value of 0.001 . The number of computational cells ranged from 32,042 for the original grid to more than 500,000 for the more developed jets. The computational time step was dynamically determined based on the estimation of the truncation error associated with the time integration scheme, resulting in time steps in the order of $\delta t \sim 10^{-3}$. The selected grid size and time step was varied in a number of runs for $\mathrm{Le}=1$ with $\mathrm{Re}=100$ to test that the results were independent of the specific values selected.

The values $\operatorname{Pr}=0.7, \alpha=0.8$ and $\beta=10$ were used for the numerical computations as representative of typical combustion conditions in realistic fuel-air mixtures. Moderately large values of the jet Reynolds number in the range $50 \leq \mathrm{Re} \leq 400$ were used, while the 
effect of the reactant diffusivity was investigated by considering Lewis numbers in the range $0.3 \leq \mathrm{Le} \leq 2$, with the lower limit being selected as representative of combustion in lean $\mathrm{H}_{2}$-air mixtures.

\subsection{Subcritical and supercritical events}

For a fixed pair of values of Re and Le, a critical value of the Damköhler number $\Delta_{c}$ was found to characterise ignition, so that for values of $\Delta$ above $\Delta_{\mathrm{c}}$ the jet discharge results in the formation of a deflagration that propagates from the jet into the surrounding ambient, consuming the available reactant, whereas for $\Delta<\Delta_{\mathrm{c}}$ the deflagration does not form, leading instead for $t \gg 1$ to a weakly reactive steady solution in which the chemical reaction is confined to the slender jet, but does not propagate to the surrounding cold reactant.

The criticality condition was determined by incrementally increasing the Damköhler number until a successful deflagration emerges, with the value of $\Delta_{\mathrm{c}}$ defined as the largest value of $\Delta$ for which ignition is not observed. For instance, in the particular case $\operatorname{Re}=100$ and Le $=1$ the critical Damköhler number was found to be $\Delta_{c}=0.76$. The flow evolution in this marginally subcritical case and also in the accompanying marginally supercritical case $\Delta=0.77$ is illustrated in Figure 1, which shows snapshots of the temperature field at different instants of time. Note that, because of the condition Le $=1$, the isocontours of temperature and those of reactant mass fraction are related according to $\Theta=1-\varphi$. Correspondingly, peak temperatures cannot in this case exceed the adiabatic flame value $\Theta=1$.
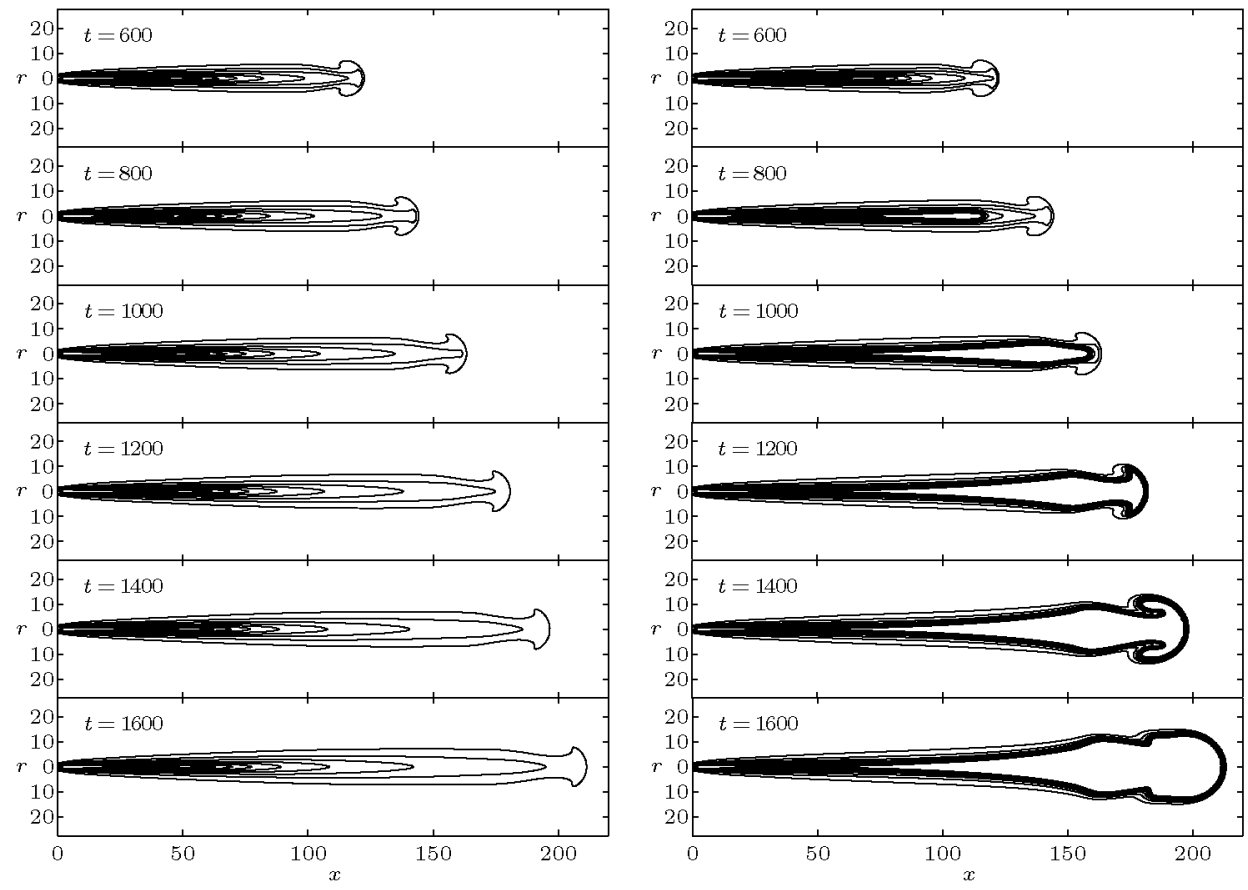

Figure 1. Isocontours of $\Theta=(0.1 / 0.9 / 0.1)$ obtained for $\operatorname{Re}=100$, Le $=1.0, \alpha=0.8$ and $\beta=10$ with $\Delta=0.76$ (left plot) and $\Delta=0.77$ (right plot). 
For $\Delta=0.76$ the products in the discharging jet mix with the outer fluid without significant chemical reaction, yielding for large times a slender weakly-reactive flow. For $\Delta=0.77$, however, a deflagration emerges. Appreciable chemical reaction is observed first in the jet stem far behind the leading vortex ring, as is clearly noticeable in the plot for $t=800$. The reactive front propagates downstream assisted by convection to reach the jet head at $t \simeq 1000$. These results suggest that the role of the leading vortex on ignition is only secondary with $\mathrm{Le}=1$, a finding further discussed below.

To understand the subsequent evolution of the flow, it is of interest to anticipate that the propagation velocity of the reactive front is in the order of the propagation velocity for the steady planar deflagration $U_{\mathrm{L}}$, whereas the axial velocity in the jet is in the order of its value at the exit, $U_{j}$. According to Equation (3), the relationship

$$
\frac{U_{\mathrm{L}}}{U_{j}}=\frac{\Delta^{1 / 2}}{\operatorname{Pr} \operatorname{Re}}
$$

holds, indicating that, for the values $\Delta \sim O(1)$ that characterise ignition, the condition $U_{\mathrm{L}} \sim \operatorname{Re}^{-1} U_{j} \ll U_{j}$ applies. As a result, the reactive front cannot propagate ahead of the jet leading edge, as can be seen in the figure for $t>1000$, but travels instead with the leading vortex once it reaches it, which occurs in this case before significant radial flame progression is observed.

On the other hand, the radial propagation of the flame into the fresh mixture surrounding the jet stem depends on the competition of the flame propagation velocity with the radial entrainment velocity induced by the jet as it develops, of order $U_{j} / \operatorname{Re} \sim U_{\mathrm{L}}$, whose value decreases inversely proportional to the radial distance. Therefore, the flame progresses radially into the surrounding atmosphere at an increasing rate. Sufficiently far from the jet axis, the axial flow velocity found is so small that the flame can propagate upstream towards the bounding wall located at $x=0$, consuming eventually all of the available reactant. These late stages of flame evolution are exhibited, for instance, in the computations shown in the lower plots of Figure 2, to be discussed below.

\subsection{Effects of reactant diffusivity}

The presence of the leading vortex continues being inconsequential for deflagration initiation when a less diffusive reactant with Le $>1$ is considered. In this case, ignition still occurs in the jet stem, at a given distance upstream from the leading vortex that increases for larger values of Le. This is illustrated in Figure 2 for $\mathrm{Le}=2$. To facilitate comparison with the results of Figure 1, the jet Reynolds number is also Re $=100$ and the conditions selected are marginally supercritical, which corresponds in this case to a Damköhler number $\Delta=7.4\left(\Delta_{\mathrm{c}}=7.3\right)$. As can be seen, a thin reactive front progresses radially from the ignition point into the cold ambient mixture, eventually propagating upstream once it reaches the slowly moving outer fluid located far from the axis. Less favourable conditions are found in this case for axial flame propagation inside the jet towards the jet head, because due to the reduced reactant diffusivity the reactant mixture found near the leading vortex is leaner. Consequently, as compared to the case $\mathrm{Le}=1$, the flame for $\mathrm{Le}=2$ reaches the leading vortex at a relatively late stage.

The sequence of events leading to ignition is fundamentally different for highly diffusive reactants with $\mathrm{Le} \ll 1$, as illustrated in Figure 3 for $\mathrm{Le}=0.3, \mathrm{Re}=100$ and $\Delta=0.035$. As in Figure 2, the case considered corresponds to marginally supercritical conditions $\left(\Delta_{c}=0.034\right)$. As can be seen, ignition occurs in this case in the well-mixed region found 

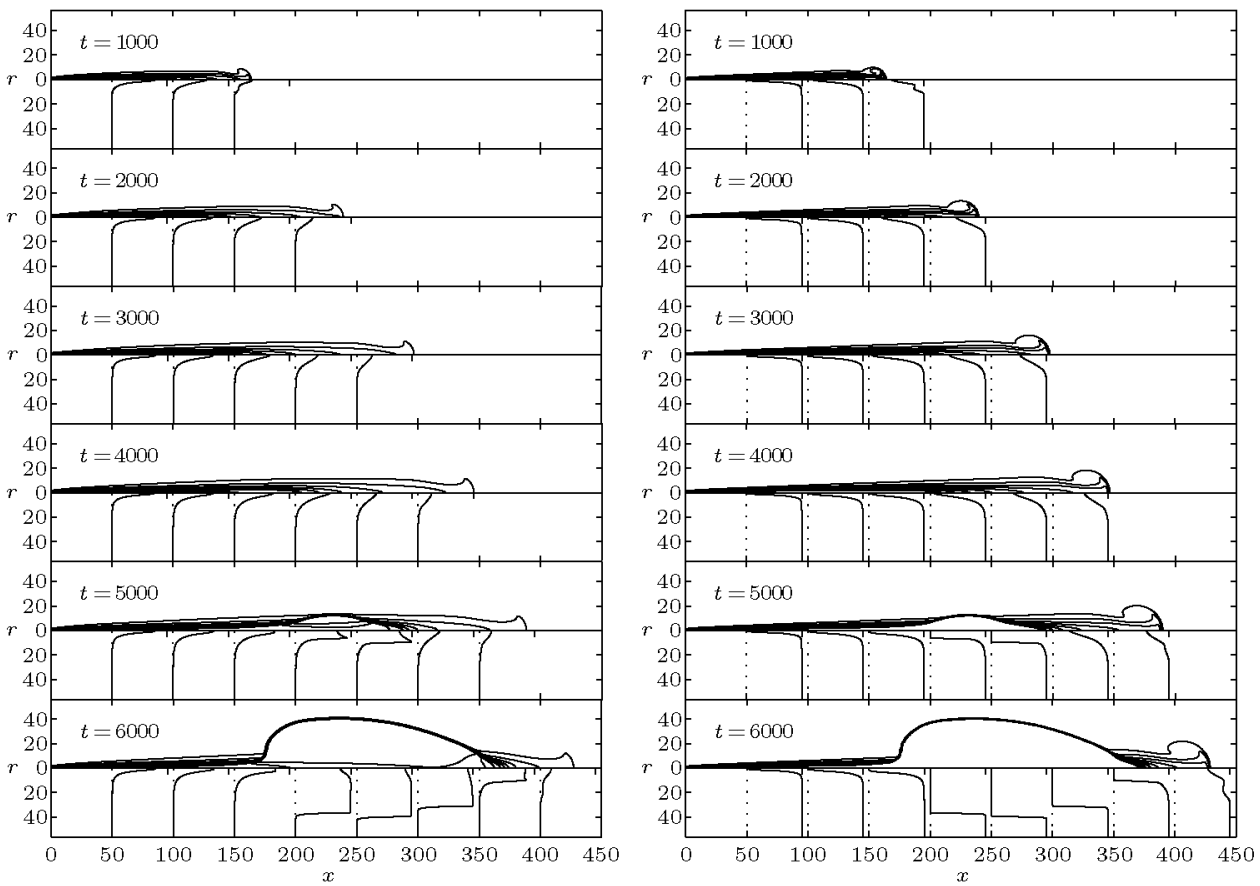

Figure 2. Isocontours and radial profiles of $\Theta=(0.1 / 0.9 / 0.1)$ (left plot) and $\varphi=(0.1 / 0.9 / 0.1)$ (right plot) obtained for $\operatorname{Re}=100, \mathrm{Le}=2.0, \alpha=0.8$ and $\beta=10$ with $\Delta=7.4$.
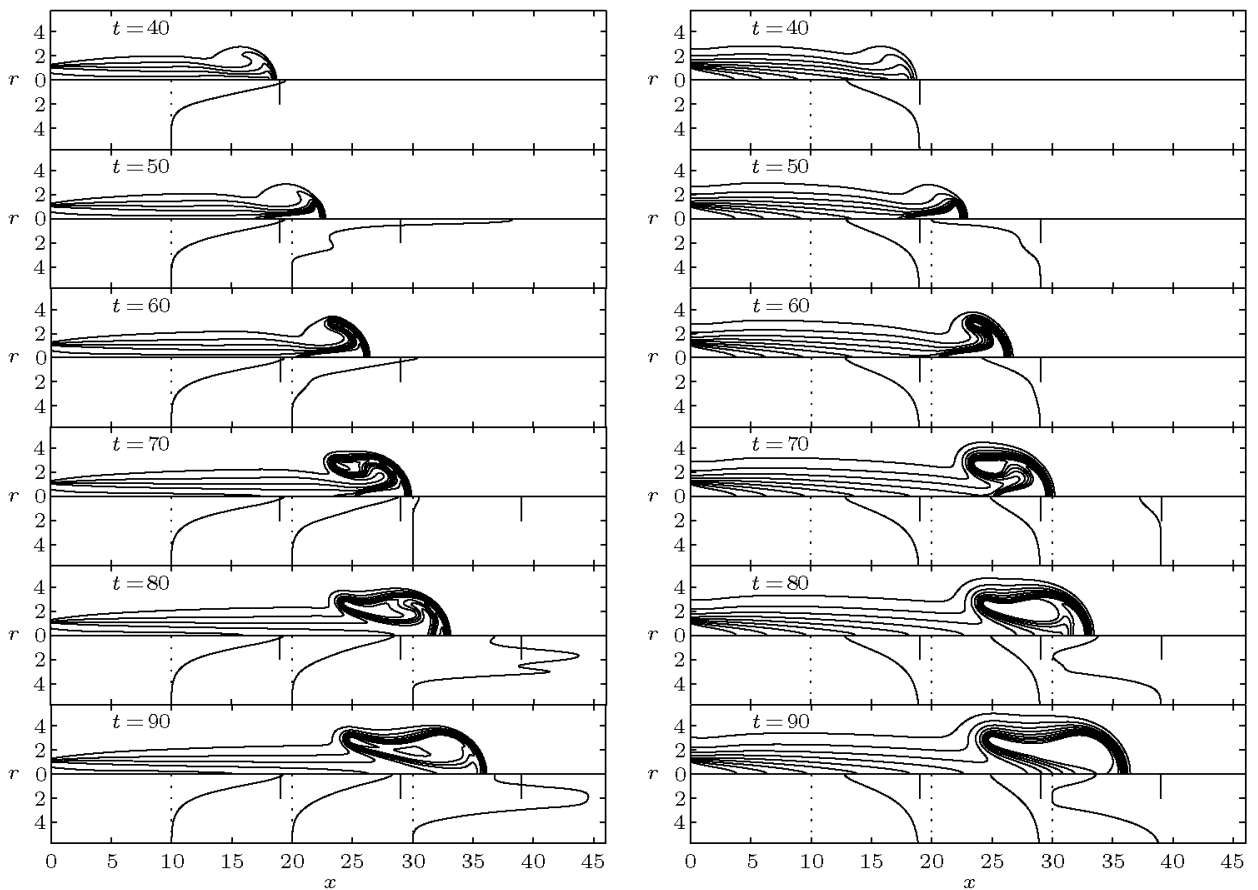

Figure 3. Isocontours and radial profiles of $\Theta=(0.2 / 1.8 / 0.2)$ (left plot) and $\varphi=(0.1 / 0.9 / 0.1)$ (right plot) obtained for $\operatorname{Re}=100, \mathrm{Le}=0.3, \alpha=0.8$ and $\beta=10$ with $\Delta=0.035$. 
at the jet head near the axis. Combustion spreads from the ignition point in the form of an annular edge flame that propagates for $50<t<70$ around the leading vortex under the influence of the induced vortical motion. The resulting edge flame, which for $t>70$ stands off at an almost constant radial distance from the axis, serves as origin for a liftoff slender cylindrical flame that develops downstream, which, at later times, is seen to propagate slowly into the surrounding reactant mixture.

In view of the critical values of the Damköhler number found for Re $=100$ as the Lewis number increases, i.e. $\Delta_{\mathrm{c}}=(0.034,0.76,7.3)$ for $\mathrm{Le}=(0.3,1.0,2.0)$, it is clear that the reactant diffusivity significantly influences the critical conditions necessary for ignition. Differential diffusion effects greatly facilitate flame initiation for $\mathrm{Le}<1$, as can be seen in the radial profiles of temperature and reactant shown in Figure 3. The reactant diffusses into the hot jet before significant heat loss to the cold surrounding ambient reduces its temperature significantly, thereby producing a preheated reactant mixture. These favourable combustion conditions lead to superadiabatic temperatures upon ignition, with peak values as large as $\Theta=2.05$ for $\mathrm{Le}=0.3$. Because of the relatively large temperature sensitivity of the chemical reaction the resulting deflagrations are initially much more robust than those found in the equidiffusional case $\mathrm{Le}=1$, and successful initiation is possible with a much smaller jet radius (i.e. much smaller values of $\Delta$ ).

The opposite behaviour is found for Le $>1$, for which the peak temperature reached after ignition lies initially below the adiabatic flame temperature $\Theta=1$, as can be observed in the radial temperature profiles of Figure 2. Heat loss towards the relatively cold fluid found near the axis hinders flame propagation into the outer reactant mixture. This heatloss effect is more pronounced for smaller values of the jet radius, so that successful flame initiation requires larger values of $a$ for Le $>1$, thereby resulting in larger values of $\Delta_{c}$.

\subsection{Influence of the Reynolds number}

The effect of the Reynolds number on flame initiation is illustrated in Figure 4, where the results shown for $\mathrm{Re}=50$ and $\mathrm{Re}=400$ are to be compared with those given previously in Figure 3 for $\mathrm{Re}=100$, the Lewis number in all cases being Le $=0.3$. In principle, the chemical time is not directly affected by changes in the jet Reynolds number. However, since the formulation employs the jet velocity and the jet radius as scales, changes in Re modify the time scale for the evolution of the temperature and mass fraction distributions as well as the resulting flame propagation velocity, as is apparent in the factor Re appearing in the conservation equations (8) and (9), and also in the denominator of Equation (14). As a result, ignition is seen to occur at later times when the Reynolds number is increased, and the emerging front propagates more slowly.

The qualitative differences that arise for increasing values of the Reynolds number are illustrated in Figure 4, where a long cylindrical flame of almost constant radius is seen to develop for $\mathrm{Re}=400$, because the radial displacement of the resulting flame is very small, while for $\mathrm{Re}=50$ the flame is seen to advance rapidly into the cold reactant mixture, moving upstream towards the orifice wall. It is worth noting that in this latter case the flame propagates initially from the ignition core with the aid of the vortical motion associated with the starting vortex, giving an evolution qualitatively similar to that found for $\operatorname{Re}=100$. The specific manner in which combustion spreads from the mixed core in this case Le $=0.3$ is however qualitatively different for $\mathrm{Re}=400$. The flame is also convected downstream by the vortical motion and is initially wrapped in the leading vortex, but the resulting annular edge flame fails to propagate along the strained mixing layer located at the leading edge of the starting vortex and does not contribute to the establishment of a cylindrical flame. The 

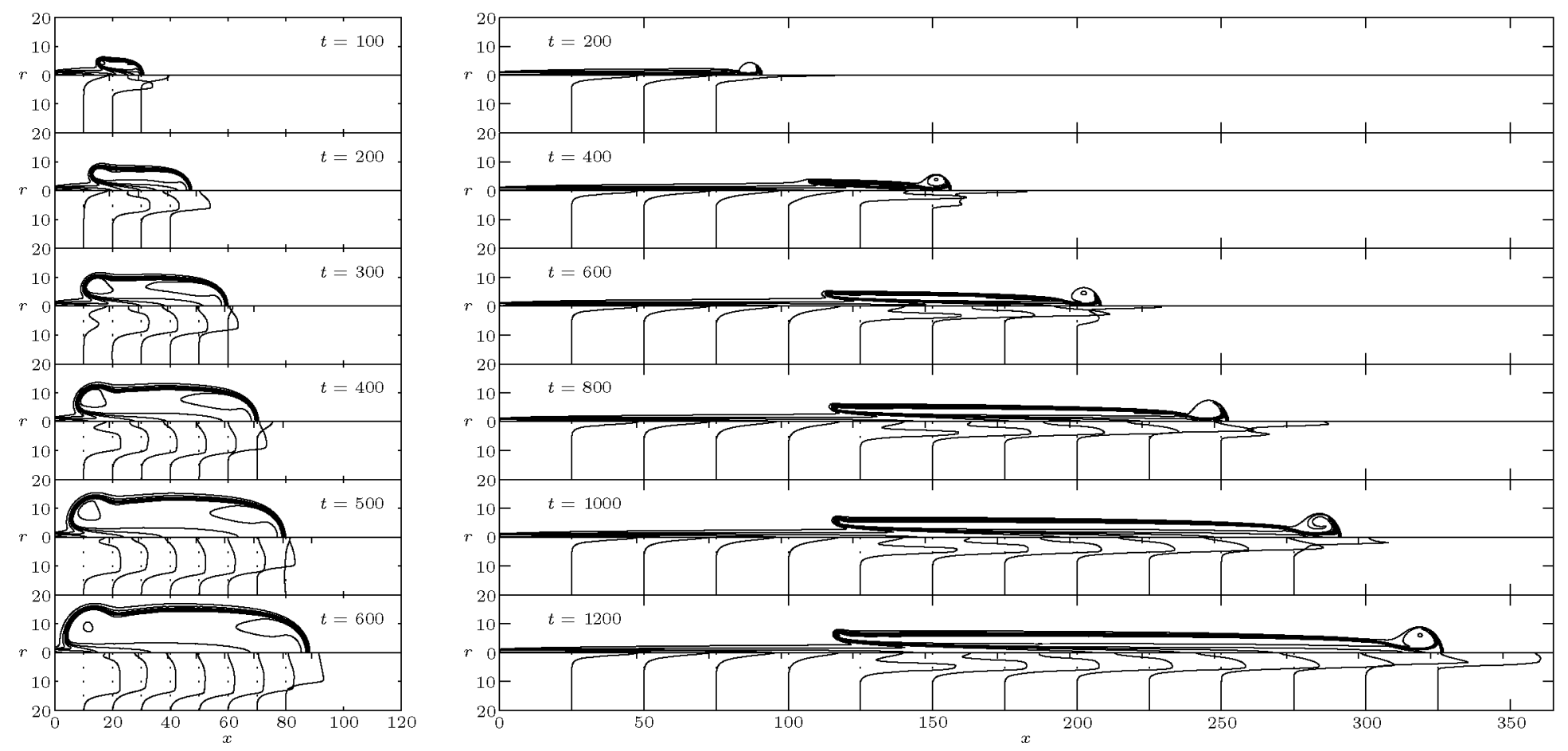

Figure 4. Isocontours and radial profiles of $\Theta=(0.2 / 1.8 / 0.2)$ obtained for $\operatorname{Le}=0.3, \alpha=0.8$ and $\beta=10$ with $\operatorname{Re}=50$ and $\Delta=0.033$ (left plot) and $\operatorname{Re}=400$ and $\Delta=0.039$ (right plot). 
standoff edge flame that appears for large times at $x \simeq 110$ develops instead from the core ignition region that lags behind the vortex near the axis.

Although ignition failures are not shown in Figures 3 and 4, it is of interest to mention that for $\operatorname{Re}=50$ and $\mathrm{Re}=100$ the marginally subcritical cases, arising for $\Delta_{\mathrm{c}}=0.032$ and $\Delta_{\mathrm{c}}=0.034$, exhibit very limited chemical activity during the jet development period, while in the case $\Delta_{\mathrm{c}}=0.038$ for $\mathrm{Re}=400$ the ignition core and the incipient flame are clearly apparent, giving up to $t=200$ temperature contours indistinguishable from those shown in the right-hand-side plots of Figure 4. At later times, however, an annular edge flame stabilised away from the axis is not observed. Instead, the reactive pocket remains trapped at the jet head and is convected downstream, causing initiation to fail.

The numerical results indicate, however, that all these subtle differences between the different ignition histories appearing for different Re seem to have only a minor effect on the resulting value of $\Delta_{c}$. As a result, the Damköhler number $\Delta_{c}$ is an appropriate parameter to characterise flame initiation in high Reynolds number jets, in that its value is almost independent of Re. In particular, for Le $=0.3$, the numerical computations yield $\Delta_{\mathrm{c}}=$ $(0.032,0.034,0.038)$ for $\mathrm{Re}=(50,100,400)$, respectively. This motivates the authors' consideration below of the boundary-layer flow arising for $\operatorname{Re} \rightarrow \infty$.

It is worth noting that for less diffusive reactants with Le $\gtrsim 1$ the influence of Re on the solution is similar to that discussed above for Le $=0.3$, in that dimensionless ignition times also increase with increasing Re and critical values $\Delta_{c}$ are only weakly affected by changes of Re. For these less diffusive fuels, ignition develops always from the jet stem, and only minor qualitative differences are observed between ignition events for different Re.

\section{Initiation criterion based on weakly-reactive steady solutions}

\subsection{Steady computations}

As seen above in Figure 1, in subcritical configurations with $\Delta<\Delta_{\mathrm{c}}$ the weakly-reactive jet that develops for $t \gg 1$ is steady and slender. The resulting solution can be described by integrating the original conservation equations (5)-(9) with the time derivative neglected in Equation (11). A critical Damköhler number $\left(\Delta_{c}\right)_{S}$ was determined as part of the steady computations, so that convergence of the integration was found to be possible only for $\Delta \leq\left(\Delta_{c}\right)_{S}$, whereas for $\Delta>\left(\Delta_{c}\right)_{S}$ a converged solution satisfying the prescribed boundary conditions could not be attained.

The resulting critical Damköhler numbers determined for $\mathrm{Le}=1$ and $\mathrm{Le}=2$ with this alternative numerical strategy are exactly equal to those found by integration of the transient jet, i.e. $\left(\Delta_{c}\right)_{S}=\Delta_{c}=(0.76,7.3)$ for Le $=(1,2)$ and $R e=100$. Full steady computations may therefore be used to estimate the critical Damköhler for reactants with Le $\gtrsim 1$. With Le $\ll 1$, however, ignition spreads from the mixed core at the transient jet head, not described in the steady computations, which therefore yield significant overpredictions for $\left(\Delta_{c}\right)_{S}>\Delta_{c}$. For instance, for Le $=0.3$ the steady computations give $\left(\Delta_{\mathrm{c}}\right)_{\mathrm{S}}=(0.44,0.047,0.045)$ for $\mathrm{Re}=(50,100,400)$, to be compared with the transient values $\Delta_{\mathrm{c}}=(0.033,0.034,0.038)$.

\subsection{Boundary-layer steady computations}

\subsubsection{The boundary-layer problem}

In the steady configuration, the hot products mix with the surrounding cold reactant in a long development region of characteristic length $x \sim$ Re across which the radial velocity 
and the transverse pressure differences take on small values of order $v \sim \operatorname{Re}^{-1}$ and $p \sim$ $\operatorname{Re}^{-2}$. The conservation equations therefore reduce, with relative errors of order $\mathrm{Re}^{-2}$, to their boundary-layer form, with axial derivatives neglected in computing the molecular transport terms and with pressure differences neglected in the momentum equation. The solution becomes independent of Re by introduction of the rescaled variables $\tilde{x}=x / \operatorname{Re}$ and $\tilde{v}=\operatorname{Re} v$. Then, the problem reduces to that of integrating the parabolic equations

$$
\begin{aligned}
\frac{\partial}{\partial \tilde{x}}(\rho u)+\frac{1}{r} \frac{\partial}{\partial r}(\rho r \tilde{v}) & =0 \\
u \frac{\partial u}{\partial \tilde{x}}+\tilde{v} \frac{\partial u}{\partial r} & =\frac{1}{\rho r} \frac{\partial}{\partial r}\left(r \mu \frac{\partial u}{\partial r}\right) \\
u \frac{\partial \varphi}{\partial \tilde{x}}+\tilde{v} \frac{\partial \varphi}{\partial r} & =\frac{1}{\operatorname{Le} \operatorname{Pr} \frac{1}{\rho r}} \frac{\partial}{\partial r}\left(r \mu \frac{\partial \varphi}{\partial r}\right)-\Omega \\
u \frac{\partial \Theta}{\partial \tilde{x}}+\tilde{v} \frac{\partial \Theta}{\partial r} & =\frac{1}{\operatorname{Pr} \frac{1}{\rho r}} \frac{\partial}{\partial r}\left(r \mu \frac{\partial \Theta}{\partial r}\right)+\Omega
\end{aligned}
$$

with initial conditions

$$
\tilde{x}=0: \quad \begin{cases}r \leq 1: & u-1=\varphi=\Theta-1=0 \\ r>1: & u=\varphi-1=\Theta=0\end{cases}
$$

and boundary conditions

$$
\tilde{x}>0: \quad\left\{\begin{array}{l}
r \rightarrow \infty: \quad u=\varphi-1=\Theta=0 \\
r=0: \quad \partial u / \partial r=\partial \varphi / \partial r=\partial \Theta / \partial r=\tilde{v}=0 .
\end{array}\right.
$$

As can be seen, for increased generality, the boundary-layer problem has been formulated by accounting for variable density $\rho=\rho^{\prime} / \rho_{\mathrm{b}}$ and variable transport coefficients, while maintaining the assumption of constant Prandtl and Lewis numbers, so that a single function $\mu=\mu^{\prime} / \mu_{\mathrm{b}}=\lambda^{\prime} / \lambda_{\mathrm{b}}=\left(\rho^{\prime} D^{\prime}\right) /\left(\rho_{\mathrm{b}} D_{\mathrm{b}}\right)$ represents the variation the viscosity $\mu^{\prime}$, thermal conductivity $\lambda^{\prime}$, and reactant diffusivity $D^{\prime}$ from their values in the burnt gas, denoted by the subscript ' $b$ '.

The problem (15)-(20) was integrated with a Crank-Nicholson scheme, using centred finite differences in the radial direction to determine the critical value of the Damköhler number corresponding to the boundary-layer approximation, $\left(\Delta_{\mathrm{c}}\right)_{\mathrm{BL}}$ for $\operatorname{Pr}=0.7$ and different values of Le, $\alpha$ and $\beta$. The jet was verified to develop correctly, in that sufficiently far downstream the velocity was seen to approach the self-similar Schlichting solution [13] with the correct value of the virtual origin [14].

\subsubsection{Constant property computations}

For comparison with the results of the full numerical computations, integrations with constant density and constant transport coefficients, i.e. values of $\rho=1$ and $\mu=1$ in Equations (15)-(18), were carried out first. Sample solutions obtained for Le $=1.0, \alpha=0.8$ and $\beta=10$ are given in Figure 5, which shows isocontours of $\varphi$ and radial temperature profiles for subcritical and supercritical values of $\Delta$. As can be seen, for $\Delta=0.85$, as the jet develops the incipient reaction remains confined to the near axis region, giving rise to 


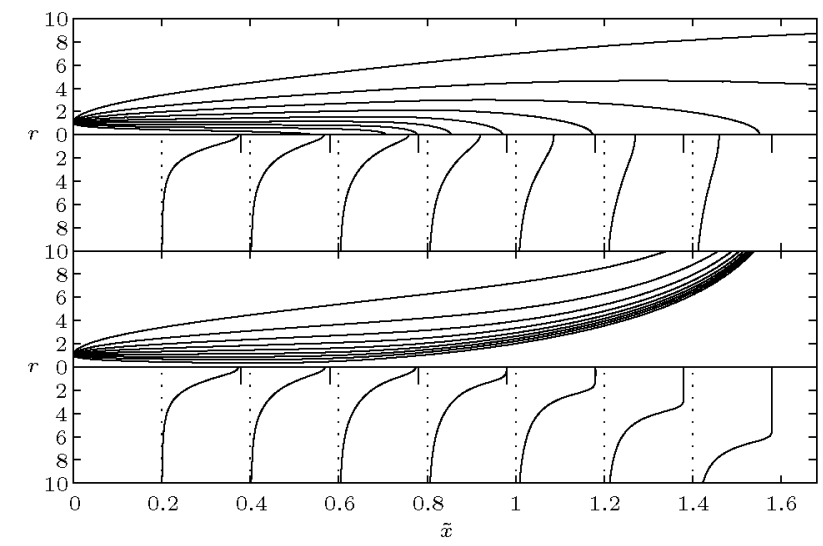

Figure 5. Isocontours of $\varphi=(0.1 / 0.9 / 0.1)$ (upper half of the plots) and the accompanying $\Theta$ profiles (lower half of the plots) obtained in the boundary-layer approximation for Le $=1.0, \alpha=0.8$ and $\beta=10$ with $\Delta=0.85$ (upper plot) and $\Delta=0.86$ (lower plot).

a slender solution with a decaying temperature along the axis. The plots for $\Delta=0.86$, on the other hand, clearly show how the chemical reaction that begins at the mixing layer separating the jet from the ambient progresses successfully to form a flame that propagates away consuming the reactant surrounding the jet. Therefore, the boundary-layer solution provides the critical Damköhler number for ignition, as the largest value of $\Delta$ for which a weakly-reactive solution emerges. Note however that, for supercritical cases with $\Delta>\left(\Delta_{\mathrm{c}}\right)_{\mathrm{BL}}$, the boundary-layer solution obtained becomes meaningless, in that as seen before in the transient computations the flame moving into the quasi-stagnant surroundings would eventually propagate upstream, consuming all the reactant available and invalidating the ambient boundary conditions stated in Equations (19) and (20).

The boundary-layer computations are the asymptotic form of the complete steady problem in the limit $\mathrm{Re} \rightarrow \infty$. Therefore, the critical Damköhler number obtained from the steady computations for increasing values of Re naturally approaches asymptotically that determined from the boundary-layer parabolic problem. For instance, for Le $=1$, the steady computations give $\left(\Delta_{c}\right)_{\mathrm{S}}=(0.76,0.79,0.81,0.82)$ for $\operatorname{Re}=(100,200,300,400)$ whereas $\left(\Delta_{\mathrm{c}}\right)_{\mathrm{BL}}=0.85$, while for $\mathrm{Le}=2$, one obtains $\left(\Delta_{\mathrm{c}}\right)_{\mathrm{S}}=(7.3,8.1,8.6)$ for $\mathrm{Re}=$ $(100,200,300)$ and $\left(\Delta_{\mathrm{c}}\right)_{\mathrm{BL}}=9.3$.

The boundary-layer formulation was also used to verify that, for the relatively large activation energies typical of combustion, the dependence of the critical Damköhler number on the specific values of $\beta$ and $\alpha$ is fairly weak. For instance, for the case $\beta=10$ and Le $=$ 1.0 considered in Figure 5 , the value of $\left(\Delta_{\mathrm{c}}\right)_{\mathrm{BL}}$ increases from $\left(\Delta_{\mathrm{c}}\right)_{\mathrm{BL}}=0.85$ to $\left(\Delta_{\mathrm{c}}\right)_{\mathrm{BL}}=$ 0.89 when $\alpha=0.8$ is replaced by $\alpha=0.9$ in the integrations. Similarly, keeping the value $\alpha=0.8$ but modifying $\beta$ also results in small changes in the critical conditions, which become for instance $\left(\Delta_{\mathrm{c}}\right)_{\mathrm{BL}}=0.82$ and $\left(\Delta_{\mathrm{c}}\right)_{\mathrm{BL}}=0.87$ for $\beta=12$ and $\beta=8$, respectively. Results of computations of critical Damköhler numbers obtained with the boundary-layer approximation for $\beta=10$ and $\alpha=0.8$ are shown in Figure 6 along with those determined previously with the complete transient and steady Navier-Stokes equations.

\subsubsection{Variable property computations}

To quantify the impact of the constant property assumption on the computed critical Damköhler numbers, variations of density and transport properties with the temperature 


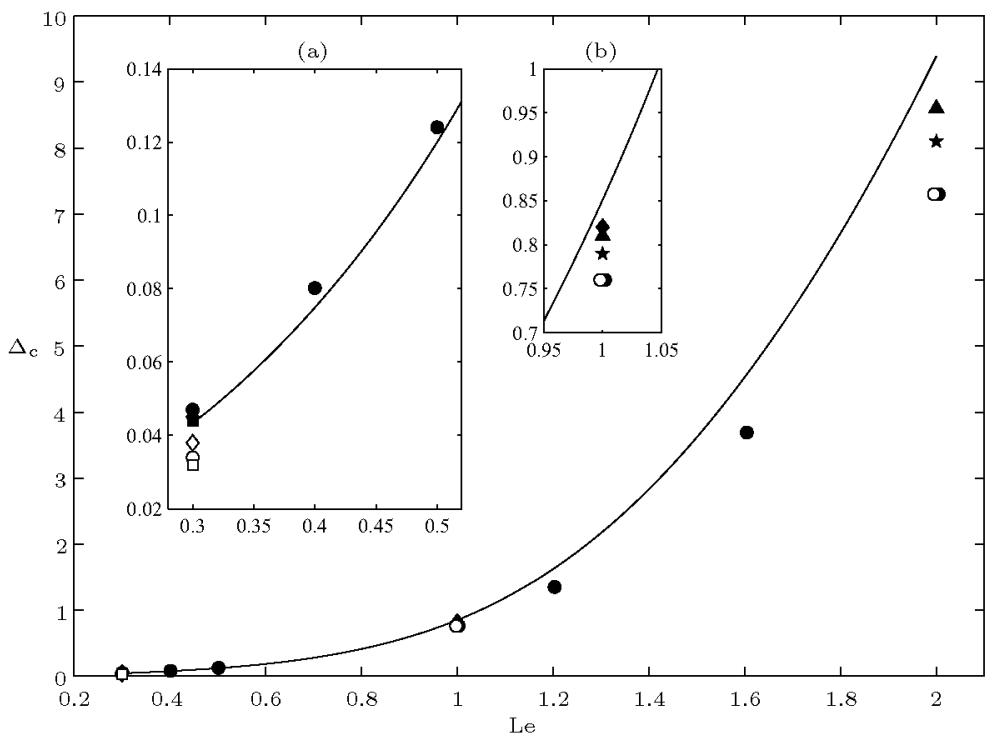

Figure 6. The variation of the critical Damköhler number with the reactant Lewis number as obtained for $\operatorname{Pr}=0.7, \alpha=0.8$ and $\beta=10$ from the boundary-layer computations (solid curve) and from transient (empty symbols) and steady (solid symbols) computations based on the Navier-Stokes equations for $\operatorname{Re}=50(\square, \boldsymbol{\square}), \operatorname{Re}=100(\circ, \bullet), \operatorname{Re}=200(\star), \operatorname{Re}=200(\boldsymbol{\Delta})$ and $\operatorname{Re}=400(\star)$. Insets (a) and (b) show closer views of the full graph.

$T=T^{\prime} / T_{\mathrm{b}}=1-\alpha(1-\Theta)$ were also incorporated in the boundary-layer computations. This was achieved by using the equation of state written in the low-Mach-number approximation with constant molecular weight $\rho=1 / T$ to compute the density and the presumed power-law $\mu=T^{\sigma}$ to calculate the temperature variation of the transport coefficients, with $\sigma=0.7$ used in the integrations. The results were both qualitatively and quantitatively similar to those obtained with $\rho=\mu=1$. In particular, differences in $\left(\Delta_{\mathrm{c}}\right)_{\mathrm{BL}}$ were found to be always relatively small. In fact, for small values of the fuel Lewis number, these differences were entirely negligible, so that, for instance, the same value $\left(\Delta_{c}\right)_{\mathrm{BL}}=0.043$ was obtained for $\mathrm{Le}=0.3$ regardless of the density and transport description employed. Differences were larger for larger values of Le, with the variable property computations giving for example $\left(\Delta_{\mathrm{c}}\right)_{\mathrm{BL}}=0.74$ and $\left(\Delta_{\mathrm{c}}\right)_{\mathrm{BL}}=7.39$ for Le $=1$ and Le $=2$, respectively, to be compared with the values $\left(\Delta_{\mathrm{c}}\right)_{\mathrm{BL}}=0.85$ and $\left(\Delta_{\mathrm{c}}\right)_{\mathrm{BL}}=9.37$ obtained with $\rho=\mu=1$. Although these quantitative findings pertain only to the boundary-layer computations, they seem to indicate that the values of $\Delta_{\mathrm{c}}$ determined from computations of transient jet flows including variations of density and transport coefficients will not be far from those determined above with the constant-density approximation, with larger departures most likely encountered for less diffusive fuels. Clearly, more computational work is required to ascertain these detailed quantitative aspects further.

\subsubsection{Connection with the hot-spot ignition problem}

As a final remark, it is worth noting that by exchanging $\varphi$ and $\Theta$ in the initial and boundary conditions, the formulation given in Equations (15)-(20) serves also to investigate ignition of a premixed jet in a hot inert environment, a problem addressed in the past in the 
planar case [15]. A connection between the problem of deflagration initiation by a hot jet and that of hot-spot ignition can also be inferred in view of the above boundary-layer formulation. To that end, consider momentarily how the problem formulation simplifies when the stagnant ambient is replaced with a coflow with velocity equal to that of the jet. In the boundary-layer approximation, the solution for the velocity reduces in that case to $u=1$. If constant density is further assumed, then $v=0$ and introduction of the time-like variable $\tau=\tilde{x} /(\mathrm{LePr})$ into Equations (17) and (18) reduces the problem to that of integrating the time dependent reaction-diffusion equations for $\varphi$ and $\Theta$ with initial conditions at $\tau=0$ given by $\Theta-1=\varphi=0$ for $r \leq 1$ and $\Theta=\varphi-1=0$ for $r>1$ and with boundary conditions for $\tau>0$ given by $\partial \Theta / \partial r=\partial \varphi / \partial r=0$ at $r=0$ and $\Theta=\varphi-1=0$ as $r \rightarrow \infty$. Clearly, the resulting simplified formulation corresponds to that required to describe ignition of a reactive mixture by a cylindrical hot pocket of reaction products, so that the classical Spalding criterion for minimum ignition energy, according to which ignition is achieved when the size of the hot spot formed is comparable to or larger than the deflagration thickness, translates in this case of hot-jet ignition in the criticality condition $a \gtrsim \delta_{\mathrm{L}}$, associated with critical Damköhler numbers of order unity.

\section{Conclusions}

The problem of deflagration initiation in a premixed fuel-air mixture by the sudden discharge of a hot jet of its adiabatic reaction products is studied numerically. The simulations are used to investigate the parametric dependence of the critical value of the Damköhler number $\left(a_{\mathrm{c}} / \delta_{\mathrm{L}}\right)^{2}$. It is found in particular that smaller values of the reactant Lewis number facilitate deflagration development because rapid reactant diffusion into the hot jet promotes superadiabatic temperatures upon ignition, thereby giving rise to robust deflagrations whose outward propagation is assisted in the first stages by the heat conducted from the products side. The reactant diffusivity also influences the manner in which ignition takes place. For reactants with molecular diffusivities in the order of or smaller than the thermal diffusivity, the flame develops as a result of ignition in the trailing mixing layer behind the leading vortex. Since unsteady mixing in the jet head plays a minor role in this case, the critical value of the orifice radius can be computed precisely by consideration of the slender weakly-reactive jet that develops for large times in subcritical cases, with the value of $a_{\mathrm{c}}$ determined from straightforward marching integration of the boundary-layer form of the steady conservation equations. By way of contrast, for highly diffusive reactants with small Lewis numbers, ignition is found to occur in the well-mixed region at the jet head, in agreement with previous experimental observations of hot-jet flame initiation events in hydrogen-air mixtures [9]. Combustion spreads from an ignition point near the axis to the leading edge of the starting vortex, where for sufficiently small values of the Reynolds number the reacting front propagates radially outward in the form of an annular edge flame under the influence of the induced vortex motion to occupy an equilibrium position at a given radial distance from the axis. This standoff edge flame serves as origin for a liftoff slender cylindrical flame that develops downstream, which, at later times, is seen to propagate slowly into the surrounding reactant. This mode of ignition changes qualitatively for sufficiently large Reynolds number, when the premixed flame is not able to survive the roll-up process and the standoff edge flame emerges instead from the core ignition region that lags behind the vortex near the axis. Because of the occurrence of ignition within the transient starting vortex, in this case of small Lewis numbers the steady computations based on the slender weakly-reactive solution tend to overpredict the critical value $a_{\mathrm{c}}$ by 
a significant amount, so that consideration of the full transient computation is needed to provide accurate results.

The value $\Delta_{c}$ can be used to compute the corresponding value of the critical orifice radius,

$$
a_{\mathrm{c}}=\frac{\lambda_{\mathrm{b}} / c_{p}}{\rho_{\mathrm{u}} U_{\mathrm{L}}} \Delta_{\mathrm{c}}^{1 / 2}
$$

above which a deflagration forms as a result of the hot-jet discharge, a quantity of relevance in connection with safety and technological applications. The solution depends on the reactant Lewis number, with a dependence displayed in Figure 6, and also on the composition, initial temperature and pressure of the specific fuel-oxidiser mixture considered, which enter into the solution through the value of the burning rate $\rho_{\mathbf{u}} U_{\mathrm{L}}$. With the chemistry model employed in the analysis, the results can be anticipated to be applicable in general to fuels whose overall combustion rate is characterised by a large temperature sensitivity. Chain-branching/chain-terminating effects, however, require consideration of a more ellaborate combustion model. For instance, the reduced mechanism developed recently for describing both high-temperature autoignition and deflagration propagation in hydrogenair mixtures [16] could be used in hydrogen-deflagration studies. For light fuels such as hydrogen, thermal diffusion, which was not considered above, is also anticipated to play a non-negligible role, promoting superadiabatic combustion through fuel diffusion into the hot jet and therefore facilitating ignition.

\section{Acknowledgements}

This work was supported by the Spanish MCINN through project numbers ENE2008-06515-C01 and CSD2010-00010 and by the Comunidad de Madrid through project number S2009/ENE-1597.

\section{References}

[1] F.A. Williams, Combustion Theory, 2nd ed., Addison-Wesley, Redwood City, CA, 1985.

[2] E. Murase, S. Ono, K. Hanada, and A.K. Oppenheim, Initiation of combustion in lean mixtures by flame jets, Combust. Sci. Technol. 113-114 (1996), pp. 167-177.

[3] M. Gharib, E. Rambod, and K. Shariff, A universal time scale for vortex ring formation, J. Fluid Mech. 360 (1998), pp. 121-140.

[4] M. Rosenfeld, E. Rambod, and M. Gharib, Circulation and formation number of laminar vortex rings, J. Fluid Mech. 376 (1998), pp. 297-318.

[5] I. Iglesias, M. Vera, A.L. Sánchez, and A. Liñán, Simulations of starting gas jets at low Mach numbers, Phys. Fluids 17 (2005), 038105. doi: 10.1063/1.1858533

[6] R. Knystautas, J.H.S. Lee, I. Moen, and H.G. Wagner, Direct initiation of a spherical detonation by a hot turbulent gas jet, Proc. Combust. Inst. 17 (1979), pp. 1235-1245.

[7] IEC 60079-1:2003(E) (International Standard), Electrical Apparatus for Explosive Gas Atmospheres - Part 1: Flameproof Enclosures "d”, International Electrotechnical Commission, Geneva, 2003.

[8] O. Larsen and R.K. Eckhoff, Critical dimensions of holes and slots for transmission of gas explosions. Some preliminary results for propane/air and cylindrical holes, J. Loss Prev. Proc. Ind. 13 (2000), pp. 341-347. 
[9] R. Sadanandan, D. Markus, R. Schiessl, U. Maas, J. Olofson, H. Seyfried, M. Richter, and M. Aldén, Detailed investigation of ignition by hot gas jets, Proc. Combust. Inst. 31 (2007), pp. 719-726.

[10] E.J. Lee and H.D. Shin, Extinction of an evolving jet flame under a linearly-varying flow field, Combust. Theory Model. 8 (2004), pp. 85-95.

[11] J. Park and H.D. Shin, Experimental investigation of the developing process of an unsteady diffusion flame, Combust. Flame 110 (1997), pp. 67-77.

[12] T. Maxworthy, Some experimental studies of vortex rings, J. Fluid Mech. 81 (1977), pp. $465-495$.

[13] H. Schlichting, Laminare strahlenausbreitung, Z. Angew. Math. Mech. 13 (1933), pp. $260-263$.

[14] A. Revuelta, A.L. Sánchez, and A. Liñán, The virtual origin as a first-order correction for the far-field description of laminar jets, Phys. Fluids 14 (2002), pp. 1821-1824.

[15] W.J. Sheu and M.C. Lin, Ignition of plane laminar premixed jets in a hot inert environment, Combust. Flame 112 (1998), pp. 285-292.

[16] P. Boivin, C. Jiménez, A.L. Sánchez, and F.A. Williams, An explicit reduced mechanism for $\mathrm{H}_{2}$-air combustion, Proc. Combust. Inst. 33 (2011), pp. 517-523. 